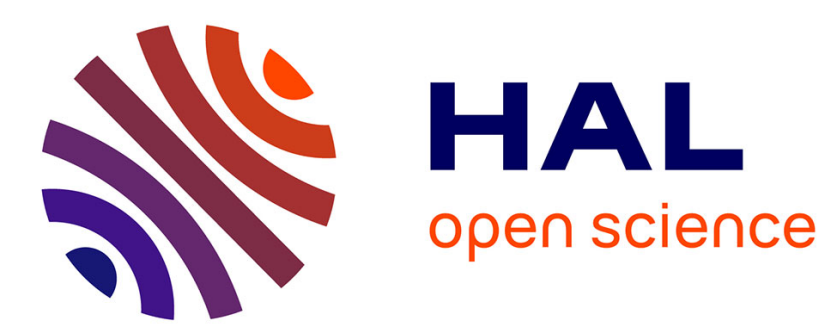

\title{
On Simulated annealing with temperature-dependent energy and temperature-dependent communication
}

\author{
Marc C. Robini, Pierre-Jean Reissman
}

\section{To cite this version:}

Marc C. Robini, Pierre-Jean Reissman. On Simulated annealing with temperature-dependent energy and temperature-dependent communication. Statistics and Probability Letters, 2011, 81 (8), pp.915. 10.1016/j.spl.2011.04.003 . hal-00753938

\section{HAL Id: hal-00753938 \\ https://hal.science/hal-00753938}

Submitted on 20 Nov 2012

HAL is a multi-disciplinary open access archive for the deposit and dissemination of scientific research documents, whether they are published or not. The documents may come from teaching and research institutions in France or abroad, or from public or private research centers.
L'archive ouverte pluridisciplinaire HAL, est destinée au dépôt et à la diffusion de documents scientifiques de niveau recherche, publiés ou non, émanant des établissements d'enseignement et de recherche français ou étrangers, des laboratoires publics ou privés. 


\section{Accepted Manuscript}

On Simulated annealing with temperature-dependent energy and

temperature-dependent communication

Marc C. Robini, Pierre-Jean Reissman

PII:

S0167-7152(11)00134-9

DOI:

10.1016/j.spl.2011.04.003

Reference: $\quad$ STAPRO 5978

To appear in: Statistics and Probability Letters

Received date: 12 January 2011

Revised date: 8 April 2011

Accepted date: 8 April 2011

Please cite this article as: Robini, M.C., Reissman, P.-J., On Simulated annealing with temperature-dependent energy and temperature-dependent communication. Statistics and Probability Letters (2011), doi:10.1016/j.spl.2011.04.003

This is a PDF file of an unedited manuscript that has been accepted for publication. As a service to our customers we are providing this early version of the manuscript. The manuscript will undergo copyediting, typesetting, and review of the resulting proof before it is published in its final form. Please note that during the production process errors may be discovered which could affect the content, and all legal disclaimers that apply to the journal pertain. 


\title{
On Simulated Annealing with Temperature-Dependent Energy and Temperature-Dependent Communication
}

\author{
Marc C. Robini ${ }^{\mathrm{a}}$, Pierre-Jean Reissman ${ }^{\mathrm{b}}$ \\ ${ }^{a}$ CREATIS (CNRS research unit UMR 5220; INSERM research unit U1044), INSA Lyon, 69621 Villeurbanne, France \\ ${ }^{b}$ Sales and e-commerce platforms division, Amadeus SAS, 485 route du Pin Montard, 06902 Sophia Antipolis, France
}

\begin{abstract}
Simulated annealing (SA) is a generic optimization method that is quite popular because of its ease of implementation and its optimal convergence properties. Still, SA is widely reported to converge very slowly and it is common practice to allow extra freedom in its design at the expense of losing global convergence guarantees.

In this paper, we derive simple sufficient conditions for the global convergence of SA when the cost function and the candidate solution generation mechanism are temperature-dependent. These conditions are surprisingly weak they do not involve the variations of the cost function with temperature - and exponential cooling makes it possible to be arbitrarily close to the best possible convergence exponent of standard SA.
\end{abstract}

Keywords: Combinatorial optimization, simulated annealing, Markov chains, Monte Carlo methods 2010 MSC: $82 \mathrm{C} 80,65 \mathrm{C} 05,60 \mathrm{~J} 10,90 \mathrm{C} 27$

\section{Introduction}

We consider the problem of finding a global minimum of an arbitrary real-valued energy function $U$ defined on a general but finite state space $E$. We denote by $U_{\text {inf }}$ the ground state energy and we let $E_{\text {inf }}(U)$ be the set of global minima of $U$, that is, $U_{\text {inf }}=\inf _{x \in E} U(x)$ and $E_{\text {inf }}(U)=\left\{x \in E \mid U(x)=U_{\text {inf }}\right\}$. A simulated annealing (SA) algorithm is a Markov chain $\left(X_{n}\right)_{n \in \mathbb{N}}$ on $E$ whose transitions are guided by a communication mechanism $q$ and controlled by a sequence of temperatures $\left(\tau_{n}\right)_{n \in \mathbb{N}^{*}}$ called a cooling schedule; the communication mechanism is a symmetric and irreducible Markov matrix on $E$ which specifies how to generate a new candidate solution from the current solution, and the cooling schedule is decreasing and converges to zero. The transitions of $\left(X_{n}\right)_{n \in \mathbb{N}}$ are defined by $\mathrm{P}\left(X_{n}=y \mid X_{n-1}=x\right)=P_{\tau_{n}}(x, y)$ with

$$
P_{\tau}(x, y)= \begin{cases}q(x, y) \exp \left(-\tau^{-1}(U(y)-U(x))^{+}\right) & \text {if } y \neq x \\ 1-\sum_{z \in E \backslash\{x\}} P_{\tau}(x, z) & \text { if } y=x,\end{cases}
$$

where $a^{+}:=\sup \{a, 0\}$. The key feature of the Markov matrix $P_{\tau}$ is that its stationary distribution $\pi_{\tau}$ tends to the uniform distribution on $E_{\text {inf }}(U)$ as the temperature $\tau$ decreases to zero $\left(\pi_{\tau}\right.$ is the Gibbs distribution with energy $U$ at temperature $\tau$ ). Consequently, the law of $X_{n}$ should stay close to $\pi_{\tau_{n}}$ if the cooling schedule decreases slowly enough, and hence we can expect that the convergence measure

$$
\mathcal{M}(n)=\sup _{x \in E} \mathrm{P}\left(X_{n} \notin E_{\text {inf }}(U) \mid X_{0}=x\right)
$$

goes to zero as $n \rightarrow+\infty$. It is well known (see Hajek (1988)) that this is the case for logarithmic cooling schedules of the form $\tau_{n}=\tau_{0} / \ln (n+1)$ provided $\tau_{0}$ is larger than the critical height $H_{U}$ of the energy landscape. Formally, $H_{U}$ is the maximum energy barrier separating a non-optimal state from a ground state, that is,

$$
H_{U}=\sup _{x \in E \backslash E_{\text {inf }}(U)} h_{U}(x)
$$


where $h_{U}(x)$ - the depth of $x$ - is defined as follows:

$$
h_{U}(x)=\inf _{y \in E_{\text {inf }}(U)} \eta_{U}(x, y)-U(x) \quad \text { with } \quad \eta_{U}(x, y)=\inf _{\left(x_{i}\right)_{i=1}^{m} \in \Pi_{q}(x, y)} \sup _{1 \leqslant i \leqslant m} U\left(x_{i}\right),
$$

where $\Pi_{q}(x, y)$ denotes the set of paths from $x$ to $y$ in the digraph

$$
(E, \Delta(q)), \quad \Delta(q)=\left\{(x, y) \in E^{2} \mid y \neq x \text { and } q(x, y)>0\right\} .
$$

Logarithmic cooling, however, is inefficient for most practical problems: $H_{U}$ is generally too large to reach the low temperature regime in a reasonable amount of computation time, while the annealing process gets easily stuck in poor local minima for feasible values of $\tau_{0}$.

The most significant advance in SA theory beyond the asymptotic properties in Hajek (1988) is due to Catoni. He showed in Catoni (1992) that exponential cooling must be preferred over logarithmic cooling when the available computing time is bounded (as is the case in practice), and that the convergence measure (2) cannot decrease faster than some optimal power of $1 / n$. More precisely, the optimal convergence speed exponent is $1 / D_{U}$, where $D_{U}-$ the difficulty of the energy landscape — is the maximum ratio of the depth to the energy level above the ground state energy:

$$
D_{U}=\sup _{x \in E \backslash E_{\mathrm{inf}}(U)} \frac{h_{U}(x)}{U(x)-U_{\mathrm{inf}}} .
$$

Besides, the upper bound $1 / D_{U}$ is sharp, since it is possible to construct a family $\left\{\left(\tau_{n}^{N}\right)_{1 \leqslant n \leqslant N} ; N \in \mathbb{N}^{*}\right\}$ of finite exponential cooling sequences such that $\ln \mathcal{M}(N) \sim \ln \left(N^{-1 / D_{U}}\right)$; such families are of the form $\tau_{n}^{N}=\tau_{0} \exp \left(-n \xi_{N}\right)$, where $\tau_{0}$ does not depend on the horizon $N$ and $\xi_{N} \sim N^{-1} \ln N$. These results provide the first theoretical justification for the widely used exponential cooling schedules. Still, although successfully applied to many difficult combinatorial optimization problems, SA is often criticized for converging very slowly. In fact, it is common practice to allow extra freedom in the design of SA algorithms, but such variations on the theme generally come without optimal convergence guarantees.

A natural generalization of SA is to allow the energy function and the communication mechanism to be temperaturedependent. We call this class of algorithms stochastic continuation (SC) by extension of the stochastic optimization processes studied in Robini et al. (2007). The first idea is to ease the annealing process by gradually revealing the complexity of the optimization problem, which is obtained by replacing the energy $U$ by the elements of a family $\left(U_{\tau}\right)_{\tau \in \mathbb{R}_{+}^{*}}$ of functions whose difficulty $D_{U_{\tau}}$ increases with decreasing $\tau$. The second idea is to facilitate the exploration of the state space by adapting the communication mechanism to the temperature regime. SC belongs to the general class of Markov processes studied in Del Moral and Miclo (1999); it includes SA with temperature-dependent energy, which is studied in Robini et al. (2007) for the finite-time case and in Frigerio and Grillo (1993) and Löwe (1996) for the asymptotic case. The convergence results in Robini et al. (2007) and Del Moral and Miclo (1999) require that

$$
\sup _{(x, \tau) \in E \times \mathbb{R}_{+}^{*}} \tau^{-1}\left|U_{\tau}(x)-U(x)\right|<+\infty,
$$

while it is assumed in Frigerio and Grillo (1993) and Löwe (1996) that there exists $a>0$ such that

$$
\sup _{(x, n) \in E \times \mathbb{N}^{*}} n^{a}\left|U_{\tau_{n}}(x)-U_{\tau_{n-1}}(x)\right|<+\infty .
$$

Both conditions (7) and (8) significantly limit the freedom in parameterizing the energy with temperature, and, in addition, the conditions for convergence in Del Moral and Miclo (1999), Frigerio and Grillo (1993) an Löwe (1996) involve impractical logarithmic cooling sequences. We show here that these limitations can be overcome while allowing the communication mechanism to vary with temperature.

The formalism and the basic ideas of SC are presented in Section 2. Our starting point for studying the convergence of SC is the observation that its transitions obey a large deviation principle with speed $\tau^{-1}$, which suggests to appeal to generalized SA (GSA) theory (see, e.g., Trouvé (1996) and Catoni (1999)). The convergence properties of GSA are outlined in Section 3, and those of SC are then derived in Section 4. Our main result states that SC with suitably adjusted exponential cooling can have a convergence speed exponent arbitrarily close to the optimal exponent $1 / D_{U}$ of SA. Moreover, the conditions for this to happen are weak and only involve the communication mechanism (putting aside the obvious necessary condition that $\left(U_{\tau}\right)_{\tau \in \mathbb{R}_{+}^{*}}$ converges pointwise to the target energy $U$ as $\tau \rightarrow 0$ ). 


\section{Definition and basic ideas}

We define an SC process with target energy landscape $(E, U, q)$ to be a family $\left(Q_{\tau}\right)_{\tau \in \mathbb{R}_{+}^{*}}$ of Markov matrices on $E$ of the form

$$
\begin{aligned}
& Q_{\tau}(x, y)= \begin{cases}q_{\tau}(x, y) \exp \left(-\tau^{-1}\left(U_{\tau}(y)-U_{\tau}(x)\right)^{+}\right) & \text {if } y \neq x \\
1-\sum_{z \in E \backslash\{x\}} Q_{\tau}(x, z) & \text { if } y=x,\end{cases} \\
& \text { with } \lim _{\tau \rightarrow 0} U_{\tau}(x)=U(x) \text { and } \quad \lim _{\tau \rightarrow 0} q_{\tau}(x, y)=q(x, y) .
\end{aligned}
$$

Given such a family together with a cooling sequence $\left(\tau_{n}\right)_{n \in \mathbb{N}^{*}}$, we call a Markov chain $\left(X_{n}\right)_{n \in \mathbb{N}}$ on $E$ with transitions $\mathrm{P}\left(X_{n}=y \mid X_{n-1}=x\right)=Q_{\tau_{n}}(x, y)$ an SC algorithm, and we denote it by $\operatorname{SC}\left(E,\left(U_{\tau}\right),\left(q_{\tau}\right),\left(\tau_{n}\right)\right)$ - we use the notation $\left(E,\left(Q_{\tau}\right)\right)$ or $\left(E,\left(U_{\tau}\right),\left(q_{\tau}\right)\right)$ for the underlying SC process. The family of energy functions $\left(U_{\tau}: E \rightarrow \mathbb{R}\right)_{\tau}$ is called the continuation scheme and the family of Markov matrices $\left(q_{\tau}: E^{2} \rightarrow[0,1]\right)_{\tau}$ is called the communication scheme. The limit communication matrix $q$ is assumed to be irreducible, as there is otherwise no guarantee to reach a ground state of the target energy $U$.

The basic idea of SC is quite easy to explain if $q_{\tau}$ is symmetric for all $\tau$ (we will relax this assumption in Section 4). Indeed, Proposition 1 below states that in this case the invariant measure $\theta_{\tau}$ of $Q_{\tau}$ is a Gibbs distribution which concentrates on the set of global minima of $U$ as $\tau \rightarrow 0$. Consequently, similarly to SA, if the cooling sequence does not decrease too fast, the law of $X_{n}$ should stay close enough to $\theta_{\tau_{n}}$ to expect convergence to an optimum.

Proposition 1. Let $\left(E,\left(Q_{\tau}\right)\right)$ be an SC process with q irreducible and $q_{\tau}$ symmetric for all $\tau$. Then, there exists $\tau^{*}>0$ such that for any $\tau \in\left(0, \tau^{*}\right], Q_{\tau}$ is irreducible and its unique invariant measure $\theta_{\tau}$ satisfies $\theta_{\tau}(x) \propto \exp \left(-\tau^{-1} U_{\tau}(x)\right)$ and $\lim _{\tau \rightarrow 0} \theta_{\tau}\left(E_{\mathrm{inf}}(U)\right)=1$.

P . For $\tau$ sufficiently small, $q_{\tau}$ inherits the irreducibility of $q$ and hence $Q_{\tau}$ is irreducible. By the symmetry of $q_{\tau}$, we have

$$
\forall(x, y) \in E^{2}, \quad \exp \left(-\tau^{-1} U_{\tau}(x)\right) Q_{\tau}(x, y)=\exp \left(-\tau^{-1} U_{\tau}(y)\right) Q_{\tau}(y, x),
$$

and therefore $\theta_{\tau}(x) \propto \exp \left(-\tau^{-1} U_{\tau}(x)\right)$.

Let $Z_{\tau}(F)=\sum_{z \in F} \exp \left(-\tau^{-1} U_{\tau}(z)\right)$ and let $\omega_{\tau}=\sup _{z \in E_{\text {inf }}(U)} U_{\tau}(z)$. We have

$$
\lim _{\tau \rightarrow 0} \frac{Z_{\tau}\left(E \backslash E_{\text {inf }}(U)\right)}{Z_{\tau}\left(E_{\text {inf }}(U)\right)}=0,
$$

and it follows that

$$
\forall x \in E, \quad \theta_{\tau}(x) \in \underset{\tau \rightarrow 0}{O}\left(\exp \left(-\tau^{-1}\left(U_{\tau}(x)-\omega_{\tau}\right)\right)\right) .
$$

Then, since $\lim _{\tau \rightarrow 0}\left(U_{\tau}(x)-\omega_{\tau}\right)=U(x)-U_{\text {inf }}$, we have $\lim _{\tau \rightarrow 0} \theta_{\tau}(x)=0$ for any $x \notin E_{\text {inf }}(U)$.

Proposition 1 gives the go-ahead for studying the global convergence properties of SC. To do so, we start from the basic observation that SC and SA behave similarly at low temperatures in the sense that

$$
\forall(x, y) \in \Delta(q), \quad \lim _{\tau \rightarrow 0}-\tau \ln Q_{\tau}(x, y)=\lim _{\tau \rightarrow 0}-\tau \ln P_{\tau}(x, y)=(U(y)-U(x))^{+} .
$$

In other words, for any $(x, y) \in E^{2}$ such that $y \neq x$ and $q(x, y)>0, Q_{\tau}(x, y)$ obeys a large deviation principle with speed $\tau^{-1}$ and rate $(U(y)-U(x))^{+}$, which suggests to appeal to the GSA theory developed in Trouvé (1996) and Catoni (1999).

\section{Generalized simulated annealing}

A GSA process on $E$ is defined by a family $\left(\Theta_{\tau}\right)_{\tau \in \mathbb{R}_{+}^{*}}$ of Markov matrices on $E$ satisfying a large deviation assumption with speed $\tau^{-1}$ and with irreducible rate function $J: E^{2} \rightarrow \mathbb{R}_{+} \cup\{+\infty\}$; that is,

$$
\forall(x, y) \in E^{2}, \quad \lim _{\tau \rightarrow 0}-\tau \ln \Theta_{\tau}(x, y)=J(x, y)
$$


(with the convention that $\ln 0=-\infty$ ), and the digraph

$$
(E, \Delta(J)), \quad \Delta(J)=\left\{(x, y) \in E^{2} \mid y \neq x \text { and } J(x, y)<+\infty\right\}
$$

is strongly connected. Given a GSA process $\left(E,\left(\Theta_{\tau}\right)\right)$ and a cooling sequence $\left(\tau_{n}\right)_{n}$, we call a Markov chain $\left(X_{n}\right)_{n \in \mathbb{N}}$ on $E$ with transitions $\mathrm{P}\left(X_{n}=y \mid X_{n-1}=x\right)=\Theta_{\tau_{n}}(x, y)$ a GSA algorithm; we denote it by $\operatorname{GSA}\left(E,\left(\Theta_{\tau}\right),\left(\tau_{n}\right)\right)$.

The Markov matrix $\Theta_{\tau}$ has a unique invariant measure $\vartheta_{\tau}$ for $\tau$ sufficiently small, and it is shown in Catoni (1999) that $\left(\vartheta_{\tau}\right)_{\tau}$ satisfies a large deviation principle: there is a function $V: E \rightarrow \mathbb{R}_{+}$, called the virtual energy, such that

$$
\forall x \in E, \quad \lim _{\tau \rightarrow 0}-\tau \ln \vartheta_{\tau}(x)=V(x)-V_{\mathrm{inf}},
$$

where $V_{\text {inf }}=\inf _{x \in E} V(x)$. Clearly, $\vartheta_{\tau}$ concentrates on the set of global minima of $V$ as $\tau \rightarrow 0$, which is to say that the virtual energy plays the role of the objective function. It is defined by

$$
\forall x \in E, \quad V(x)=\inf _{T \in \mathcal{T}(x)} \sum_{(z, t) \in \mathcal{E}_{T}} J(z, t),
$$

where $\mathcal{T}(x)$ is the set of directed trees $T=\left(E, \mathcal{E}_{T}\right), \mathcal{E}_{T} \subset E^{2}$, with root $x$ and whose edges are directed towards $x$ (i.e., $d^{+}(x)=0$ and $d^{+}(y)=1$ for all $y \neq x$, where $d^{+}(z)$ is the outdegree of vertex $z$ in $\left.T\right)$. Similarly to standard SA theory, the triplet $(E, V, J)$ defines an energy landscape and is accompanied with a critical depth $H_{V}$ and a difficulty $D_{V}$; these two constants are defined by

$$
H_{V}=\sup _{x \in E \backslash E_{\text {inf }}(V)} h_{V}(x) \quad \text { and } \quad D_{V}=\sup _{x \in E \backslash E_{\text {inf }}(V)} \frac{h_{V}(x)}{V(x)-V_{\text {inf }}},
$$

where $h_{V}(x)$ denotes the depth of $x$ in $(E, V, J)$ :

$$
h_{V}(x)=\inf _{y \in E_{\text {inf }}(V)} \eta_{V}(x, y)-V(x) \quad \text { with } \quad \eta_{V}(x, y)=\inf _{\left(x_{i}\right)_{i=1}^{m} \in \Pi_{J}(x, y)} \sup _{1 \leqslant i \leqslant m-1}\left(V\left(x_{i}\right)+J\left(x_{i}, x_{i+1}\right)\right),
$$

where $\Pi_{J}(x, y)$ is the set of paths from $x$ to $y$ in $(E, \Delta(J))$.

The main convergence result for GSA is stated in the following theorem; it gives an asymptotic bound on the probability of error for suitably adjusted piecewise-constant exponential cooling sequences.

Theorem 1 (Catoni (1999)). Let $\left(E,\left(\Theta_{\tau}\right)\right)$ be a GSA process with virtual energy landscape $(E, V, J)$. For any positive reals $\alpha, \beta$ and $\gamma$, consider the family of finite-time algorithms $\left\{\left(X_{n}\right)_{0 \leqslant n \leqslant \sigma K}=\operatorname{GSA}\left(E,\left(\Theta_{\tau}\right),\left(\tau_{n}^{\sigma, K}\right)\right) ; \sigma, K \in \mathbb{N}^{*}\right\}$ with cooling sequence of the form

$$
\tau_{n}^{\sigma, K}=\frac{1}{\alpha \ln K}\left(\frac{\gamma}{\beta}\right)^{\frac{1}{\sigma}\left\lfloor\frac{n-1}{K}\right\rfloor}
$$

If $\alpha<1 / H_{V}, \beta>H_{V} / D_{V}$ and $\gamma<\beta$, then, for any $\sigma$,

$$
\liminf _{K \rightarrow+\infty}-\frac{\ln \sup _{x \in E} \mathrm{P}\left(V\left(X_{\sigma K}\right) \geqslant V_{\text {inf }}+\gamma \mid X_{0}=x\right)}{\ln (\sigma K)} \geqslant \frac{1}{D_{V}}\left(\frac{\gamma}{\beta}\right)^{\frac{1}{\sigma}} .
$$

\section{Finite-time convergence of stochastic continuation}

To apply Theorem 1 to optimization by SC, we need to find sufficient conditions for an SC process with target energy $U$ to be a GSA process with virtual energy $V$ such that $E_{\text {inf }}(V) \subset E_{\text {inf }}(U)$. This is the subject of the two propositions below. The conditions for an SC process to fit into the GSA framework only involve the communication scheme; they are given in Proposition 2. Proposition 3 gives a simple additional condition for the virtual energy to be equal to the target energy plus a constant. 
Proposition 2. Let $\left(E,\left(Q_{\tau}\right)\right)$ be an SC process with communication scheme $\left(q_{\tau}\right)_{\tau}$ and with target energy landscape $(E, U, q)$. Assume that

(A1) $q$ is irreducible,

(A2) $\forall(x, y) \in E^{2}, q(x, y)=0 \Longrightarrow \lim _{\tau \rightarrow 0}\left(q_{\tau}(x, y)\right)^{\tau}=\mathbf{1}_{\{x=y\}}$.

Then $\left(E,\left(Q_{\tau}\right)\right)$ is a GSA process with rate function

$$
J(x, y)= \begin{cases}(U(y)-U(x))^{+} & \text {if } q(x, y)>0 \text { or } x=y \\ +\infty & \text { otherwise }\end{cases}
$$

$\mathrm{P} \quad$. For any $(x, y) \in E^{2}$,

$$
\begin{aligned}
(x \neq y \text { and } q(x, y)>0) & \Longrightarrow-\tau \ln Q_{\tau}(x, y)=-\tau \ln q_{\tau}(x, y)+(U(y)-U(x))^{+} \underset{t \rightarrow 0}{\longrightarrow}(U(y)-U(x))^{+}, \\
(x \neq y \text { and } q(x, y)=0) & \Longrightarrow-\tau \ln Q_{\tau}(x, y) \geqslant-\tau \ln q_{\tau}(x, y) \underset{t \rightarrow 0}{\longrightarrow}+\infty, \\
x=y & \Longrightarrow 0 \leqslant-\tau \ln Q_{\tau}(x, x) \leqslant-\tau \ln \left(1-\sum_{z \neq x} q_{\tau}(x, z)\right)=-\tau \ln q_{\tau}(x, x) \underset{t \rightarrow 0}{\longrightarrow} 0 .
\end{aligned}
$$

The irreducibility of $J$ follows from the irreducibility of $q$.

Proposition 3. Let $\left(E,\left(Q_{\tau}\right)\right)$ be an $S C$ process with target energy landscape $(E, U, q)$ and satisfying assumptions (A1) and (A2). If

(A3) $\Delta(q)$ is symmetric (i.e., $\left.\forall(x, y) \in E^{2}, q(x, y)>0 \Longleftrightarrow q(y, x)>0\right)$,

then the virtual energy $V$ of $\left(E,\left(Q_{\tau}\right)\right)$ satisfies $V-V_{\mathrm{inf}}=U-U_{\mathrm{inf}}$.

$\mathrm{P} \quad$. Let $(x, y) \in E^{2}$ such that $x \neq y$. Let $T \in \mathcal{T}(x)$ and let $\pi_{T}(x, y)=\left(x_{i}\right)_{i=1}^{m}$ be the (unique) path from $x$ to $y$ in $T$. Assume that $\pi_{T}(x, y)$ is $J$-admissible, that is, $J\left(x_{i}, x_{i+1}\right)<+\infty$ for all $i \in\{1, \ldots, m-1\}$. Then, by (19) and (A3), $J\left(x_{i}, x_{i+1}\right)-J\left(x_{i+1}, x_{i}\right)=U\left(x_{i+1}\right)-U\left(x_{i}\right)$ for all $i$, and it follows that

$$
\sum_{(z, t) \in \mathcal{E}_{T}: z \in \pi_{T}(x, y)} J(z, t)=U(x)-U(y)+\sum_{(z, t) \in \mathcal{E}_{T}: z \in \pi_{T}(x, y)} J(t, z) .
$$

Since $J$ is irreducible, there exists $T \in \mathcal{T}(x)$ such that $J(T):=\sum_{(z, t) \in \mathcal{E}_{T}} J(z, t)<+\infty$ (and hence such that $\pi_{T}(x, y)$ is $J$-admissible); consequently, using (20),

$$
V(x)+U(y)=\inf _{T \in \mathcal{T}(x): J(T)<+\infty}\left(\sum_{(z, t) \in \mathcal{E}_{T}: z \notin \pi_{T}(x, y)} J(z, t)+\sum_{(z, t) \in \mathcal{E}_{T}: z \in \pi_{T}(x, y)} J(t, z)+U(x)\right) .
$$

Letting $\zeta: \mathcal{T}(x) \rightarrow \mathcal{T}(y)$ be the one-to-one mapping that reverses the orientation of each edge in the path from $y$ to $x$, we obtain

$$
V(x)+U(y)=\inf _{T \in \mathcal{T}(x): J(\zeta(T))<+\infty} J(\zeta(T))+U(x)=V(y)+U(x) .
$$

In particular, given $y_{0} \in E_{\text {inf }}(U)$, we have $V(x)=V\left(y_{0}\right)+U(x)-U_{\text {inf }} \geqslant V\left(y_{0}\right)$ for all $x$, and hence $V\left(y_{0}\right)=V_{\text {inf }}$.

Our main result is given by Theorem 2. Putting it simply, it states that increasing the number of temperature stages of piecewise-constant exponential cooling makes it possible for SC to have a convergence speed exponent arbitrarily close to the optimal convergence speed exponent of SA.

Theorem 2. Let $\left(E,\left(U_{\tau}\right),\left(q_{\tau}\right)\right)$ be an SC process with target energy landscape $(E, U, q)$ and satisfying assumptions (A1)-(A3), and let $\mathcal{M}$ be the convergence measure defined in (2). For any $\varepsilon>0$, there is a family of piecewise-constant cooling sequences $\left(\tau_{n}^{\sigma, K}\right)_{1 \leqslant n \leqslant \sigma K}$ with $\sigma$ stages of length $K$ such that the family of finite-time algorithms

$$
\left\{\left(X_{n}\right)_{0 \leqslant n \leqslant \sigma K}=\operatorname{SC}\left(E,\left(U_{\tau}\right),\left(q_{\tau}\right),\left(\tau_{n}^{\sigma, K}\right)\right) ; \sigma, K \in \mathbb{N}^{*}\right\}
$$


satisfies

$$
\lim _{K \rightarrow+\infty}-\frac{\ln \mathcal{M}(\sigma K)}{\ln (\sigma K)} \geqslant \frac{1}{(1+\varepsilon) D_{U}}
$$

These cooling sequences are of the form

$$
\tau_{n}^{\sigma, K}=A_{K} \exp \left(-\frac{B}{\sigma}\left\lfloor\frac{n-1}{K}\right\rfloor\right)
$$

with $A_{K}>\frac{H_{U}}{\ln K}, \quad B>\ln \left(\frac{H_{U}}{D_{U} \inf _{x \in E \backslash E_{\text {inf }}(U)} U(x)-U_{\text {inf }}}\right)$, and $\sigma \geqslant \frac{B}{\ln (1+\varepsilon)}$.

$\mathrm{P}$. Let $(E, V, J)$ be the virtual energy landscape associated with $\left(E,\left(U_{\tau}\right),\left(q_{\tau}\right)\right)$. From Propositions 2 and 3 , we have $E_{\mathrm{inf}}(V)=E_{\mathrm{inf}}(U), \Delta(J)=\Delta(q)$, and for any $(z, t) \in \Delta(J)$,

$$
V(z)+J(z, t)=U(z)-U_{\mathrm{inf}}+V_{\mathrm{inf}}+(U(t)-U(z))^{+}=\sup \{U(z), U(t)\}-U_{\mathrm{inf}}+V_{\mathrm{inf}} .
$$

It follows that $\eta_{V}-V_{\text {inf }}=\eta_{U}-U_{\text {inf }}$, and thus $h_{V}=h_{U}, H_{V}=H_{U}$, and $D_{V}=D_{U}$. Consequently, for a family of finite-time SC algorithms of type (21) with cooling sequence of the form (17), Theorem 1 gives that if $\alpha<1 / H_{U}$, $\beta>H_{U} / D_{U}$, and $\gamma<\beta$, then

$$
\forall \varepsilon>0, \quad \forall \sigma \geqslant \frac{\ln (\beta / \gamma)}{\ln (1+\varepsilon)}, \quad \liminf _{K \rightarrow+\infty}-\frac{\ln \sup _{x \in E} \mathrm{P}\left(U\left(X_{\sigma K}\right) \geqslant U_{\text {inf }}+\gamma \mid X_{0}=x\right)}{\ln (\sigma K)} \geqslant \frac{1}{(1+\varepsilon) D_{U}} .
$$

Taking $\gamma<\inf _{x \in E \backslash E_{\text {inf }}(U)} U(x)-U_{\text {inf }}$ gives the asymptotic bound in (22).

The conditions for Theorem 2 to apply are weak; they only concern the communication scheme $\left(q_{\tau}\right)_{\tau}$. Assumptions (A1) and (A3) are standard in SA theory: the irreducibility of the limit $q$ and the symmetry of its support $\Delta(q)$ ensure that the target energy landscape can be fully explored and that any path in this landscape can be traveled in the opposite direction. The meaning of (A2) is less clear, but a simple sufficient condition for it to hold is that $q(x, x)>0$ for all $x$ and that $\Delta\left(q_{\tau}\right)=\Delta(q)$ for $\tau$ small enough. In other words, it suffices to allow the limit communication mechanism to rest anywhere and to "freeze" the set of possible moves at low temperatures. Quite interestingly, there is no condition on the continuation scheme $\left(U_{\beta}\right)_{\beta}$ apart from pointwise convergence to the target energy.

\section{Acknowledgements}

This work was partly supported by the French National Research Agency under grant ANR-09-BLAN-0372-01. The authors would like to thank the referee for helpful comments and suggestions.

\section{References}

Catoni, O., 1992. Rough large deviation estimates for simulated annealing: application to exponential schedules. Ann. Probab. 20 (3), $1109-1146$. Catoni, O., 1999. Simulated annealing algorithms and Markov chains with rare transitions. In: Séminaire de probabilités XXXIII. Vol. 1709 of Lecture Notes in Math. Springer, New York, pp. 69-119.

Del Moral, P., Miclo, L., 1999. On the convergence and applications of generalized simulated annealing. SIAM J. Control Optim. 37 (4), $1222-$ 1250.

Frigerio, A., Grillo, G., 1993. Simulated annealing with time-dependent energy function. Math. Z. 213, 97-116.

Hajek, B., 1988. Cooling schedules for optimal annealing. Math. Oper. Res. 13 (2), 311-329.

Löwe, M., 1996. Simulated annealing with time-dependent energy function via Sobolev inequalities. Stochastic Process. Appl. 63 (2), $221-233$

Robini, M. C., Lachal, A., Magnin, I. E., 2007. A stochastic continuation approach to piecewise constant reconstruction. IEEE Trans. Image Process. 16 (10), 2576-2589.

Trouvé, A., 1996. Cycle decompositions and simulated annealing. SIAM J. Control Optim. 34 (3), 966-986. 